\title{
Nota metodológica \\ sobre los indicadores \\ del barómetro del CIS
}

Pilar Rey del Castillo

RESUMEN

Recientemente, un conjunto de indicadores confeccionados a base de los barómetros de opinión mensuales están apareciendo en la página web del Centro de Investigaciones Sociológicas (Centre for Sociological Research). Este trabajo describe la metodología empleada para su construcción, pero antes de adentrarse en este tema muestra otros indicadores similares confeccionados en diferentes instituciones.

Palabras clave: Encuestas por Correo, Encuestas Telefónicas, Marco Poblacional, No Cobertura. 


\section{INTRODUCCIÓN}

Los barómetros del Centro de Investigaciones Sociológicas (CIS) son las encuestas de opinión que, con periodicidad mensual, se realizan para hacer el seguimiento de las opiniones y actitudes de la sociedad española ante distintas situaciones o acontecimientos de la actualidad.

La mayoría de las preguntas que incluye la encuesta de cada mes se refieren a cuestiones de la actualidad más estricta (congreso del partido del gobierno, atentado del 11 de marzo...) o a un tema que se elige como central (expectativas para el año próximo, cultura política,...$)$ y sólo una pequeña parte son preguntas que se repiten a lo largo del tiempo, con periodicidades más o menos fijas. Como la metodología de los barómetros es similar en lo que se refiere al ámbito, universo, procedimiento de muestreo, tamaño de la muestra, etc., las respuestas a estas preguntas son comparables y dan lugar a las correspondientes series temporales.

De esta forma, se encuentran en los barómetros series de preguntas, mensuales o trimestrales, relativas a la evaluación de la situación política actual y de su evolución futura, la evaluación de la situación económica actual y de su evolución futura, la evaluación de la labor del gobierno de la nación y del primer partido de la oposición, la intención de voto en elecciones generales, la estimación de voto y la autoubicación ideológica en una escala de graduación. Desde 1996 estas preguntas se mantienen de una forma casi continua con una formulación similar, por lo cual ésta va a ser la fecha a partir de la cual vamos a estudiar y construir los indicadores, aunque se tengan resultados para periodos anteriores.

Aunque las respuestas a algunas de estas preguntas constituyen, en sí mismas, indicadores de la evolución de ciertas variables, a menudo resulta interesante tratar de resumir varias preguntas que se refieren a un mismo tema en una serie temporal o indicador que sintetice en una única cifra el conjunto de la información en cada momento del tiempo. También, para una única pregunta con varias opciones de respuesta, puede resultar de interés la construcción de un indicador sintético.

En el próximo apartado se presentan las metodologías para la construcción de algunos de los indicadores de temas sociales más conocidos. A continuación se presenta y justifica el tipo de indicador seleccionado. En los últimos apartados se muestran en detalle las metodologías para cada una de las series. 


\section{METODOLOGÍAS PARA LA CONSTRUCCIÓN DE INDICADORES}

Los números índices constituyen una rama tradicional de la estadística que estudia la construcción de indicadores o índices para comparar en el espacio o en el tiempo los fenómenos económicos y sociales.

Cuando se trata de fenómenos que son medibles a través de una variable cuantitativa, como puede ser el volumen de la producción, el nivel de los precios, etc., la literatura ofrece toda una serie de posibilidades: los índices simples, índices compuestos de Laspeyres o de Paasche, índice ideal de Fisher, etc.

En el caso de las encuestas de opinión, las variables a las que dan lugar las preguntas suelen ser variables cualitativas, con distintas opciones de respuesta. Así, por ejemplo, la pregunta:

Comparando la responsabilidad de los centros educativos con la de la familia, en lo que se refiere al desarrollo social y personal de los jóvenes, ¿cree Ud. que...?

\begin{tabular}{lr} 
& $\%$ \\
Los centros deberían tener la responsabilidad principal & 2,8 \\
La responsabilidad debería ser compartida & 63,7 \\
La familia debería tener la responsabilidad principal & 32,2 \\
NS & 1,1 \\
NC & 0,2 \\
\hline TOTAL & 100,0
\end{tabular}

da lugar a una variable cualitativa con cinco opciones de respuesta. Los resultados se presentan, en general, como los porcentajes de respuesta para cada una de las opciones.

Para este tipo de variables no sirven los índices tradicionales y ha de tratar de construirse algún indicador alternativo que sintetice en una sola cifra toda la información. Esto suele hacerse, por analogía con el caso de las variables cuantitativas, calculando números índices, saldos de respuestas, etc.

A continuación se presentan algunos de los indicadores más conocidos que se construyen a partir de encuestas de opinión en el ámbito internacional. 
- Índice de Confianza del Consumidor. Este indicador es elaborado desde 1967 para The Conference Board en Estados Unidos. Los cuestionarios se envían por correo a una muestra representativa de 5.000 familias (responden unas 3.500 en promedio), diferente cada mes. Incluyen 5 preguntas:

1. Valoración de la situación económica actual.

2. Expectativas sobre la situación económica dentro de seis meses.

3. Valoración de la situación de empleo actual.

4. Expectativas sobre la situación de empleo dentro de seis meses.

5. Expectativas sobre el total de los ingresos familiares dentro de seis meses.

Para cada una de las preguntas hay tres opciones de respuesta: positiva, negativa o neutra.

Para cada pregunta, el número de respuestas positivas se divide entre la suma de las positivas y las negativas para obtener el valor relativo o proporción. Estas proporciones de respuesta son ajustadas estacionalmente y, posteriormente, divididas por la proporción media del año 1985 para obtener un índice con base 1985 de cada pregunta.

Se obtienen los indicadores:

— Índice de Confianza del Consumidor: promedio de los 5 índices.

— Índice de situación actual: promedio de los índices de las preguntas 1 y 3.

— Índice de expectativas: promedio de los índices de las preguntas 2, 4 y 5.

- Índice de Confort del Consumidor. La revista $A B C$ News/Money elabora este indicador a partir de una encuesta telefónica mensual de ámbito nacional (EE.UU.) a alrededor de 1.000 adultos desde 1985 .

Las tres preguntas utilizadas son:

1. ¿Describiría usted la situación actual de la economía nacional como excelente, buena, no tan buena o pobre? 
2. ¿Describiría usted la situación actual de sus finanzas personales como excelente, buena, no tan buena o pobre?

3. Considerando el coste actual de las cosas y sus finanzas personales propias, ¿diría usted que el momento actual es excelente, bueno, no tan bueno o pobre para comprar las cosas que quiere y necesita?

Se calculan los saldos de respuesta (diferencia entre las respuestas positivas y las negativas) para cada pregunta. Posteriormente se suman estos 3 saldos y se dividen entre 3 para obtener el Índice de Confort del Consumidor. Por tanto, este indicador tiene un rango entre +100 (todo respuestas positivas en las 3 preguntas) y -100 (todo respuestas negativas en las 3 preguntas).

- Índice de Sentimiento del Consumidor. El Centro de Investigación del Consumo de la Universidad de Michigan realiza una encuesta mensual a los consumidores a partir de la cual se obtienen los indicadores.

Las cinco preguntas incluidas son:

1. $\left(x_{1}\right)$ Estamos interesados en la situación financiera actual de las personas. ¿Diría que usted (y la familia con la que convive) está financieramente mejor o peor que hace un año?

2. $\left(x_{2}\right)$ Mirando adelante, ¿cree que dentro de un año usted (y la familia con la que convive) estará financieramente mejor, peor o igual que ahora?

3. $\left(x_{3}\right)$ Volviendo a las condiciones económicas del país en conjunto, ¿cree que durante los próximos doce meses tendremos financieramente buenos tiempos, malos tiempos $u$ otra cosa?

4. $\left(x_{4}\right)$ Mirando adelante, ¿diría usted que es más esperable que en el país tengamos durante los próximos cinco años continuamente buenos tiempos o tengamos desempleo generalizado, depresión u otra cosa?

5. $\left(x_{5}\right)$ Respecto a las cosas que la gente compra para sus hogares - tales como muebles, una nevera, televisión y cosas como éstas-, hablando en general, ¿cree que ahora es un buen o un mal momento para que la gente compre grandes objetos para el hogar?

Para calcular el Índice de Sentimiento del Consumidor (ICS) se calculan primero las puntuaciones relativas (el porcentaje de respuestas positivas menos el porcentaje de respues- 
tas negativas más 100) para cada una de las 5 preguntas. Después se redondean al número entero más próximo. Usando la fórmula que se muestra a continuación, se suman las 5 puntuaciones relativas, se divide por la puntuación relativa del año base 1996, que es 6,7558 , y se añade 2,0 (una constante que trata de corregir cambios en el diseño de la muestra de los años cincuenta):

$$
\operatorname{ICS}=\frac{x_{1}+x_{2}+x_{3}+x_{4}+x_{5}}{6,7558}+2,0
$$

Usando el mismo procedimiento, se calculan el Índice de Situación Económica Actual (ICC) y el Índice de Expectativas del Consumidor (ICE) según las siguientes fórmulas:

$$
\text { ICC }=\frac{x_{1}+x_{5}}{2,6424}+2,0 \quad, \quad I C E=\frac{x_{2}+x_{3}+x_{4}}{4,1134}+2,0
$$

- Índice de Optimismo Económico. Elaborado por el TechnoMetrica Institute of Policy and Politics (TIPP) de Estados Unidos, se basa en las respuestas a una encuesta telefónica mensual dirigida a aproximadamente 1.000 adultos. Para este indicador, las preguntas son:

1. En los próximos seis meses, ¿piensa que la situación económica en el país será mejor, peor o similar a la actual?

2. En los próximos seis meses, ¿piensa que su situación económica personal será mejor, peor o similar a la actual?

3. ¿Cómo se considera respecto a las políticas económicas federales actuales para mantener la economía hacia la buena dirección: muy satisfecho, algo satisfecho, no muy satisfecho o en absoluto satisfecho?

El cálculo se hace de la siguiente forma:

- Si la pregunta tiene una respuesta positiva y una negativa (mejor y peor, aprueba y desaprueba), el componente del índice para esa pregunta es el siguiente:

$$
K=50+0,5^{*}\left(p_{+}-p_{-}\right)
$$

donde $p_{+}$es el porcentaje de respuesta positiva y $p_{-}$el porcentaje de respuesta negativa. 
- Si la pregunta admite una selección entre dos respuestas positivas y dos negativas (muy satisfecho, algo satisfecho, no muy satisfecho, en absoluto satisfecho), el componente del índice utiliza la siguiente fórmula:

$$
K=50+0,5^{*}\left(p_{++}-p_{--}\right)+0,25^{*}\left(p_{+}-p_{-}\right)
$$

donde $p_{++}$es el porcentaje que responde fuertemente positivo, $p_{--}$es el porcentaje que responde fuertemente negativo, $p_{+}$es el porcentaje que responde moderadamente positivo y $p_{-}$el porcentaje que responde moderadamente negativo.

Posteriormente, se hace un promedio de los componentes de las preguntas para calcular el índice agregado. Cuando el nivel de un índice es superior a 50, el número de respuestas positivas es superior al número de respuestas negativas. Si todas las respuestas son fuertemente positivas el índice es 100, y si todas son fuertemente negativas el índice es 0 .

- Índice de Liderazgo Presidencial. Elaborado también por el TIPP, se basa en una encuesta telefónica mensual análoga. Para este indicador, las preguntas son:

1. En conjunto, ¿su opinión acerca de George W. Bush es generalmente favorable, generalmente no favorable o no le es lo suficientemente familiar para opinar?

2. En general, ¿aprueba o desaprueba la forma en la que George W. Bush realiza su trabajo como presidente o no le es lo suficientemente familiar para opinar?

3. ¿Cómo describiría el liderazgo que el Presidente Bush está proporcionando para el país? ¿Diría que es muy fuerte, fuerte, moderado, débil o muy débil?

El cálculo de los índices se hace de la misma forma que el anterior.

- Índice de Perspectiva Nacional. Elaborado también por el TIPP, también se basa en una análoga encuesta telefónica mensual. Sus preguntas son:

1. En general, ¿cómo de satisfecho está usted con la dirección que las cosas tienen en el país en este momento? ¿Diría que está muy satisfecho, algo satisfecho, no muy satisfecho o en absoluto satisfecho?

2. Hablando en general, ¿cómo de satisfecho está usted con la dirección que las cosas tienen en el país en este momento en términos morales y éticos? ¿Diría que está muy satisfecho, algo satisfecho, no muy satisfecho o en absoluto satisfecho? 
3. Hablando en general, en los próximos seis meses, ¿piensa que la posición de Estados Unidos como líder mundial será mejor, peor o igual que la que es ahora?

4. En los próximos seis meses, ¿piensa que su calidad de vida será mejor, peor o igual que la que es ahora?

También el cálculo de los índices se hace como los anteriores.

\section{CÁLCULO DE LOS INDICADORES DEL BARÓMETRO}

Como ya se ha indicado, los porcentajes de respuesta directa a las preguntas constituyen en sí mismos, a veces, indicadores de los fenómenos que describen. Un ejemplo válido serían los porcentajes de intención de voto a cada partido político. Para estos casos no es necesaria la construcción de indicadores adicionales.

En este apartado vamos a describir el procedimiento general de cálculo que utilizaremos para aquellas preguntas en las que resulta de interés la construcción del indicador. Vamos a verlo para el caso de una pregunta aislada, ya que, como se ha observado en el apartado anterior, es en esto en lo que varían los distintos métodos. A la hora de agregar los resultados de varias preguntas, en todos los casos se toma, como indicador para el conjunto, la media aritmética de los indicadores para cada pregunta.

Comparando los métodos de cálculo mostrados en el anterior apartado, se pueden hacer algunas observaciones:

- Los índices calculados para el Índice de Confort del Consumidor toman valores entre -100 y 100. Éstos son más difíciles de interpretar que los que toman sólo valores positivos y resultan especialmente complicados cuando se quieren interpretar todos respecto a un mismo periodo base.

- En los casos en que existen tres opciones de respuesta (positiva, nula y negativa) con proporciones de respuesta $p_{+}, p_{0}$ y $p_{-}$, respectivamente, se suelen tomar los saldos de respuesta por coincidir con la media de la variable:

$$
\text { Media }=1 \cdot p_{+}+0 \cdot p_{0}+(-1) \cdot p_{-}=p_{+}+p_{-}
$$

- El Índice de Confianza del Consumidor tiene como periodo base el año 1985, y el Índice de Sentimiento del Consumidor tiene base 1996. En la teoría de números índices, el objeti- 
vo al tomar un periodo base es poner las cifras en una misma magnitud, para que se puedan comparar y operar entre ellas. Es decir, cuando se tienen medidas en alguna magnitud física para cada periodo (por ejemplo, volúmenes de producción en kilos, litros...), se divide por la magnitud del periodo base, para obtener unas cifras - las proporciones respecto al periodo base-que son comparables.

En el caso que nos ocupa - las respuestas a preguntas que dan origen a variables cualitativas - los resultados se toman en forma de proporciones de respuesta para cada categoría, cifras que ya son comparables directamente, por lo que no parece necesario tomar un periodo base (posteriormente, siempre se pueden hacer todos los índices relativos a un periodo base sin más que dividirlos por la media aritmética de ese periodo).

Por otra parte, la elección del periodo base permite una cierta manipulación que puede afectar a la validez de los índices. Como ejemplo, véanse en el grafico 1 las series de puntuaciones entre 0 y 100 Serie1 y Serie2 desde el año 2000 al 2002. Estas puntuaciones son directamente comparables y se observa que en el 2002 toman ambas el mismo valor de 50.

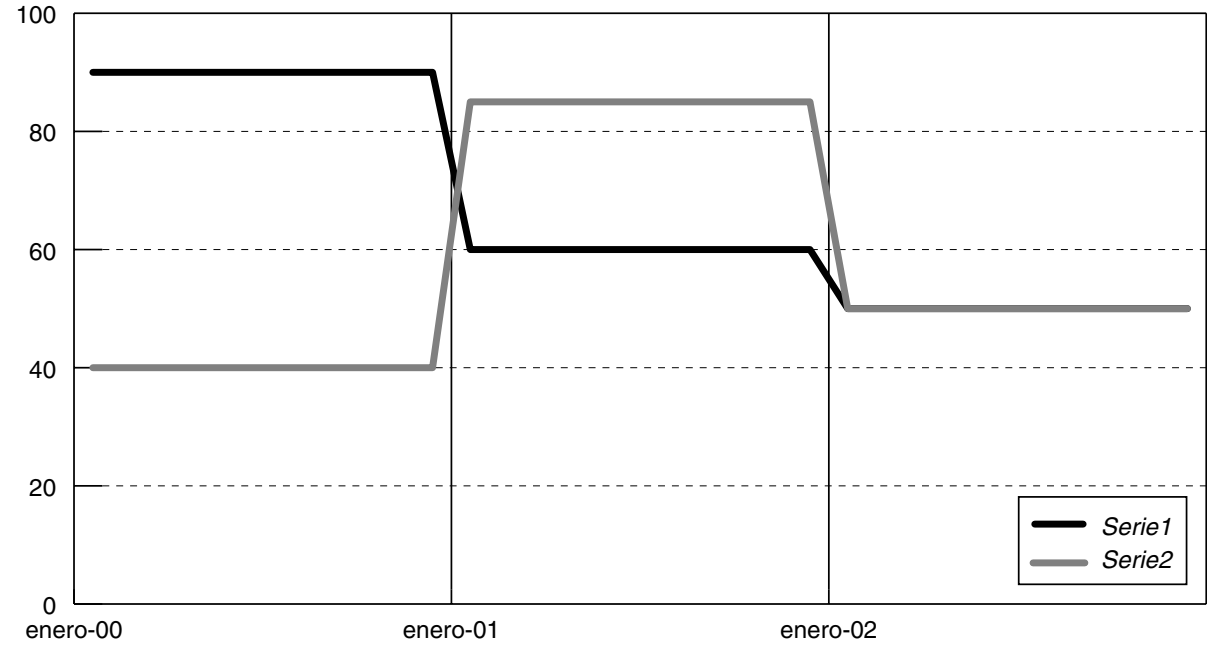

Podemos tomar como base el año 2000 y se obtienen las series que se presentan en el gráfico 2. 
GRÁFICO 2

Base 2000

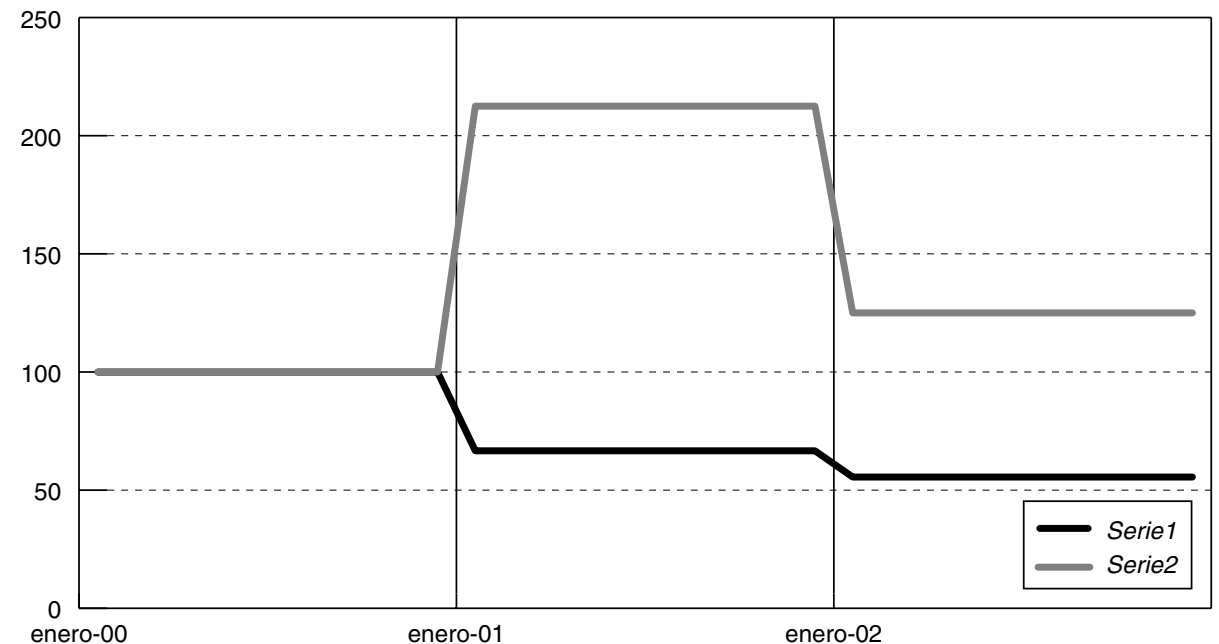

Pero también podríamos tomar como año base el 2001, obteniéndose el grafico 3.

GRÁFICO 3

Base 2001

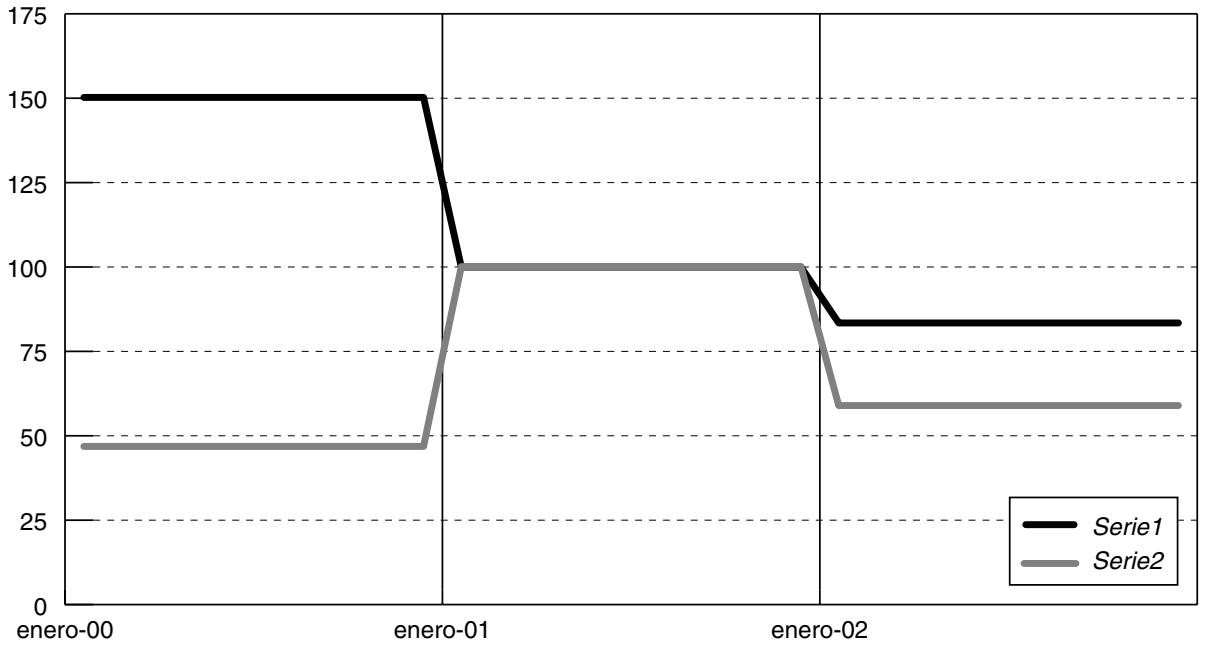


Es decir, podemos elegir un año base que nos permita ver más positivamente en el año 2002 a la Serie1 o la Serie2, según convenga, a pesar de que las puntuaciones para ese año son idénticas. Para evitar estas flexibilidades, parece más razonable, en cifras que son comparables directamente, no tomar un periodo base fijo.

- El Índice de Confianza del Consumidor de The Conference Board desestacionaliza previamente las series de las proporciones de cada opción de respuesta. Sin embargo, las proporciones de respuesta de las series de los barómetros del CIS analizadas no tienen componente estacional significativo, por lo que no parece necesario añadir este paso, que supone una mayor complejidad en la elaboración de los indicadores. Por otra parte, a la hora de construir indicadores agregados, es discutible si es preferible desestacionalizar y luego agregar los indicadores o proceder al revés.

Visto lo anterior, tratamos de construir indicadores sin los inconvenientes señalados y, a la vez, lo más sencillos posible.

Las preguntas para las que queremos construir indicadores son las que tienen opciones de respuesta del tipo:

- Positivo

- Nulo

- Negativo

o del tipo:

- Muy bueno

- Bueno

- Regular

- Malo

- Muy malo

es decir, opciones de respuesta en las que se observa una graduación. Por este motivo, se puede asociar a cada opción de respuesta una puntuación. Elegimos puntuaciones entre 0 y 100 desde lo más negativo a lo más positivo, por su facilidad de utilización. Otorgamos estas puntuaciones desde un punto de vista bayesiano, donde no hay ninguna razón que nos aleje de suponer la equidistancia. Por tanto, para el caso de 5 opciones de respuesta, tendremos: 


\begin{tabular}{llr} 
& Opción & Puntuación \\
\hline$x_{1}$ & Muy bueno & 100 \\
\hline$x_{2}$ & Bueno & 75 \\
\hline$x_{3}$ & Regular & 50 \\
\hline$x_{4}$ & Malo & 25 \\
\hline$x_{5}$ & Muy malo & 0
\end{tabular}

y para el caso de tres opciones:

\begin{tabular}{llr} 
& Opción & Puntuación \\
\hline$x_{1}$ & Positivo & 100 \\
\hline$x_{2}$ & Nulo & 50 \\
\hline$x_{3}$ & Negativo & 0
\end{tabular}

El indicador que elegimos es la media aritmética de las puntuaciones ponderada con las proporciones de respuesta. Así, para el caso de 5 opciones, si $p_{1}, p_{2}, p_{3}, p_{4}$ y $p_{5}$ son las proporciones de respuesta respectivas de las distintas opciones, el indicador será:

$$
I=\frac{\sum_{i=1}^{5} p_{i} \cdot x_{i}}{\sum_{i=1}^{5} p_{i}}
$$

El cálculo sería análogo en el caso de tener tres opciones o en el caso de no tener categoría u opción de respuesta central.

Este indicador parece el «natural» en variables que presentan graduación en las opciones de respuesta y tiene la ventaja de su sencillez.

Además, coincide en su cálculo con otros indicadores cuya construcción es, en apariencia, más sofisticada: los índices de Optimismo Económico, Liderazgo Presidencial y Perspectiva Nacional elaborados por el TIPP.

En efecto, si tenemos 5 opciones de respuesta, el cálculo del TIPP sería: 


$$
\begin{aligned}
K & =50+0,5^{\star}\left(p_{++}-p_{--}\right)+0,25^{\star}\left(p_{+}-p_{-}\right)= \\
& =50+0,5^{\star}\left(\frac{p_{1}}{\sum_{i=1}^{5} p_{i}} \cdot 100-\frac{p_{5}}{\sum_{i=1}^{5} p_{i}} \cdot 100\right)+0,25^{\star}\left(\frac{p_{2}}{\sum_{i=1}^{5} p_{i}} \cdot 100-\frac{p_{4}}{\sum_{i=1}^{5} p_{i}} \cdot 100\right)= \\
& =\frac{50 \cdot\left(p_{1}+p_{2}+p_{3}+p_{4}+p_{5}\right)+50 p_{1}-50 p_{5}+25 p_{2}-25 p_{4}}{\sum_{i=1}^{5} p_{i}}= \\
& =\frac{100 p_{1}+75 p_{2}+50 p_{3}+25 p_{4}+0 p_{5}}{\sum_{i=1}^{5} p_{i}}=\frac{\sum_{i=1}^{5} x_{i} \cdot p_{i}}{\sum_{i=1}^{5} p_{i}}=I
\end{aligned}
$$

Un aspecto a tener en cuenta al construir los indicadores del CIS es el hecho de que, en este caso, no se trata de diseñar un cuestionario con las preguntas que se consideren idóneas para ello, sino que se trata de aprovechar las preguntas que se han venido haciendo durante años con metodología similar. Por ello, a veces no se construyen los indicadores que se podrían considerar los mejores.

Pasamos a describir en los próximos apartados todos los índices e indicadores que se elaboran a partir de los barómetros. Se presentan agrupados por temas, tal como se construyen.

\section{INDICADORES DE LA SITUACIÓN ECONÓMICA}

En los barómetros de todos los meses (excepto en agosto, que no se elabora la encuesta) aparecen dos preguntas relativas a la situación económica:

P1: Refiriéndonos ahora a la situación económica general de España, ¿cómo la calificaría Ud.: muy buena, buena, regular, mala o muy mala?

P2: ¿Y cree Ud. que dentro de un año la situación económica del país será mejor, igual o peor que ahora?

A veces se pregunta también por la situación retrospectiva, pero, al no haberse realizado desde 1996 de forma sistemática, no se considera para la elaboración de los indicadores. 
Se construye el Indicador de la Situación Económica Actual (SEA) a partir de la pregunta P1 como:

$$
S E A=\frac{100 \cdot p_{1}+75 \cdot p_{2}+50 \cdot p_{3}+25 \cdot p_{4}+0 \cdot p_{5}}{p_{1}+p_{2}+p_{3}+p_{4}+p_{5}}
$$

donde $p_{1}, p_{2}, p_{3}, p_{4}$ y $p_{5}$ son, respectivamente, las proporciones de respuesta de las opciones muy buena, buena, regular, mala y muy mala, respectivamente.

Análogamente, se construye el Indicador de Expectativas Económicas (IEE) a partir de la pregunta P2 como:

$$
\text { IEE } \frac{100 \cdot p_{1}+50 \cdot p_{2}+0 \cdot p_{3}}{p_{1}+p_{2}+p_{3}}
$$

donde $p_{1}, p_{2}$ y $p_{3}$ son, respectivamente, las proporciones de respuesta de las opciones mejor, igual y peor, respectivamente.

Por último, el Indicador de Confianza Económica (ICE) es la media aritmética de los otros dos:

$$
I C E=\frac{S E A+I E E}{2}
$$

En los gráficos 4 a 7 se muestran estos indicadores desde 1996. 
GRÁFICO 4

Indicador de Confianza Económica (ICE)

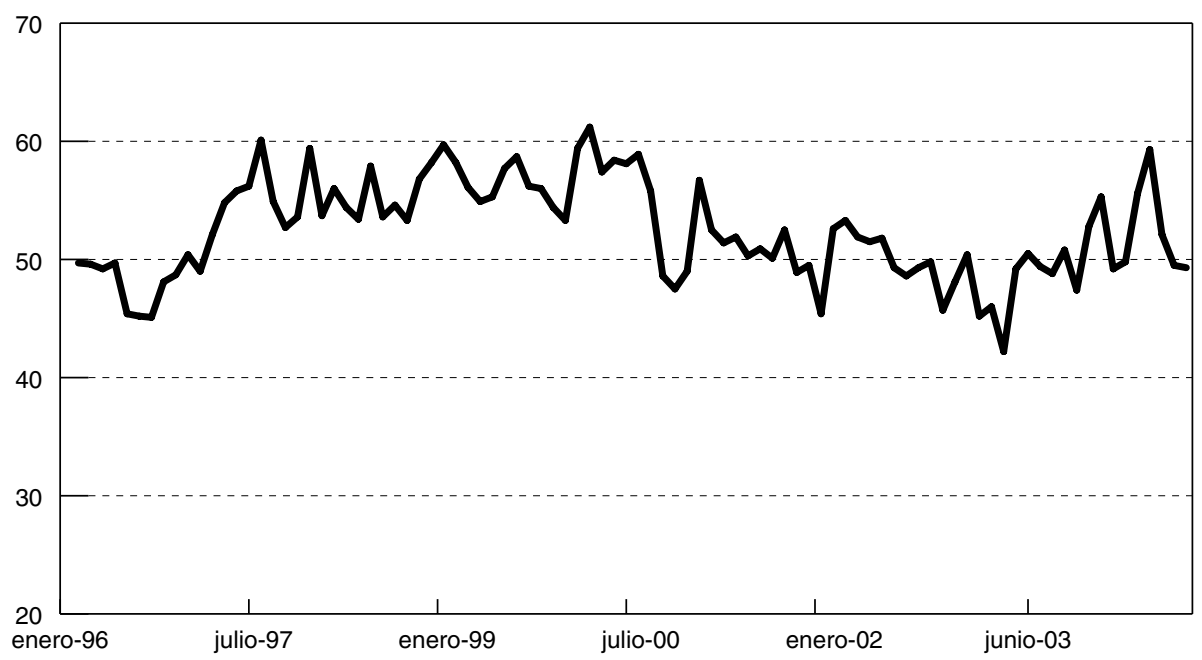

GRÁFICO 5

Indicador Situación Económica Actual (SEA)

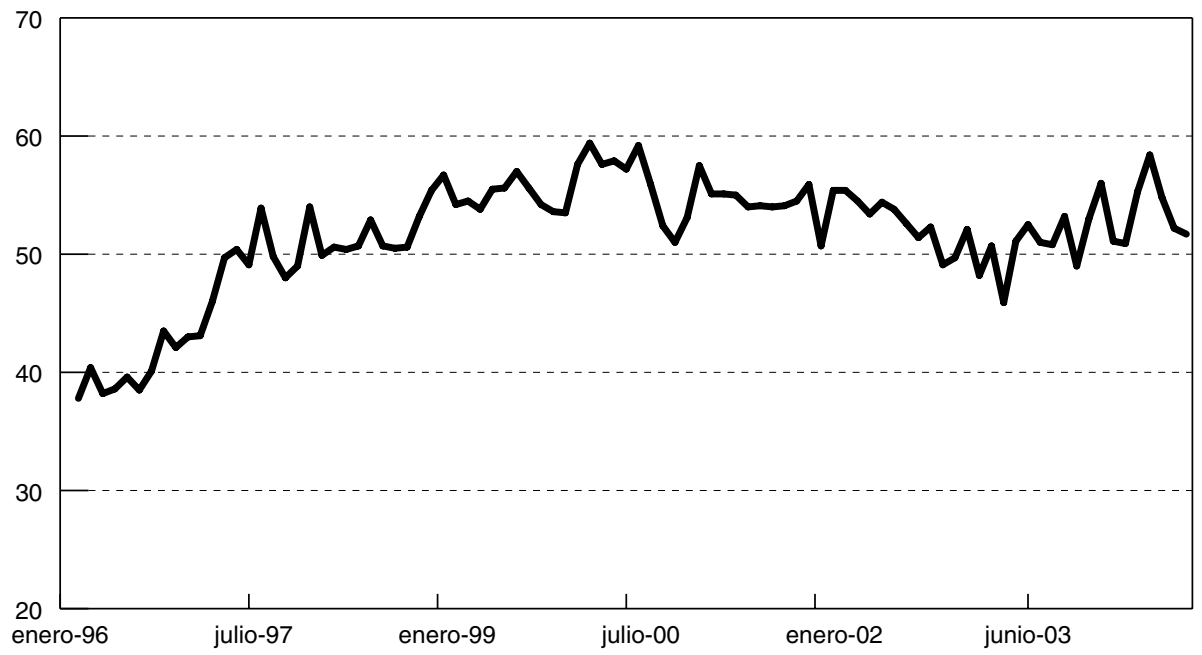


GRÁFICO 6

Indicador Expectativas Económicas (IEE)

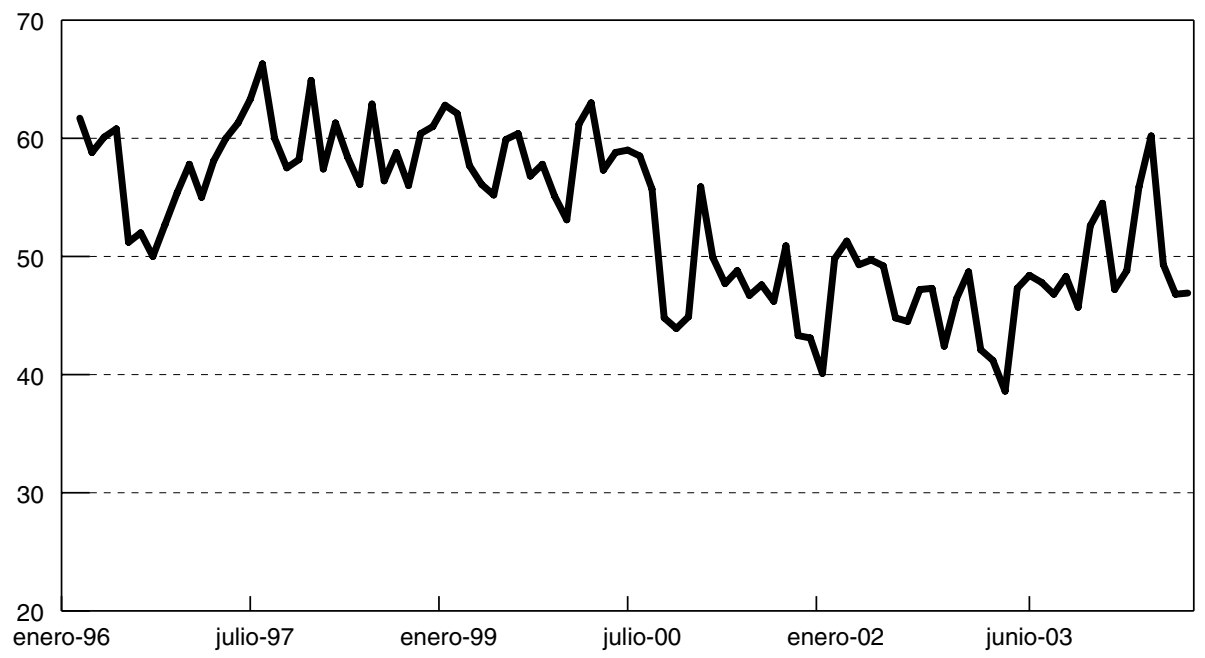

GRÁFICO 7

Indicadores de Situación Económica

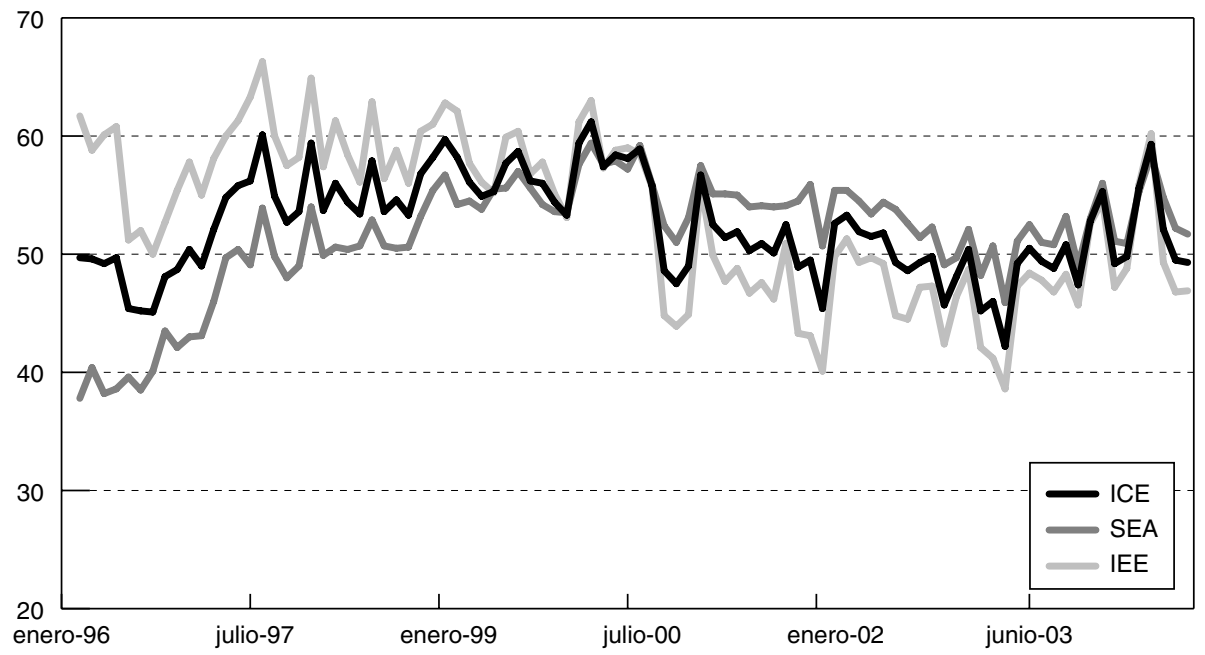




\section{INDICADORES DE LA SITUACIÓN POLÍTICA}

Las preguntas de los barómetros de todos los meses relativas a la situación política son:

P1: Refiriéndonos ahora a la situación política general de España, ¿cómo la calificaría Ud.: muy buena, buena, regular, mala o muy mala?

P2: ¿Y cree Ud. que dentro de un año la situación política del país será mejor, igual o peor que ahora?

Es decir, son preguntas totalmente análogas a las relativas a la situación económica. También se pregunta, a veces, por la situación retrospectiva, pero, al no haberse realizado desde 1996 de forma sistemática, no se considera para la elaboración de los indicadores. Éstos se construirán en forma análoga.

El Indicador de la Situación Política Actual (SPA) a partir de la pregunta P1 será:

$$
S P A=\frac{100 \cdot p_{1}+75 \cdot p_{2}+50 \cdot p_{3}+25 \cdot p_{4}+0 \cdot p_{5}}{p_{1}+p_{2}+p_{3}+p_{4}+p_{5}}
$$

donde $p_{1}, p_{2}, p_{3}, p_{4}$ y $p_{5}$ son, respectivamente, las proporciones de respuesta de las opciones muy buena, buena, regular, mala y muy mala, respectivamente.

El Indicador de Expectativas Políticas (IEP) a partir de la pregunta P2 será:

$$
I E P=\frac{100 \cdot p_{1}+50 \cdot p_{2}+0 \cdot p_{3}}{p_{1}+p_{2}+p_{3}}
$$

donde $p_{1}, p_{2}$ y $p_{3}$ son, respectivamente, las proporciones de respuesta de las opciones mejor, igual y peor, respectivamente.

Por último, el Indicador de Confianza Política (ICP) es la media aritmética de los otros dos:

$$
I C P=\frac{S P A+I E P}{2}
$$

En los gráficos 8 a 11 se muestran estos indicadores. 
GRÁFICO 8

Indicador de Confianza Política (ICP)

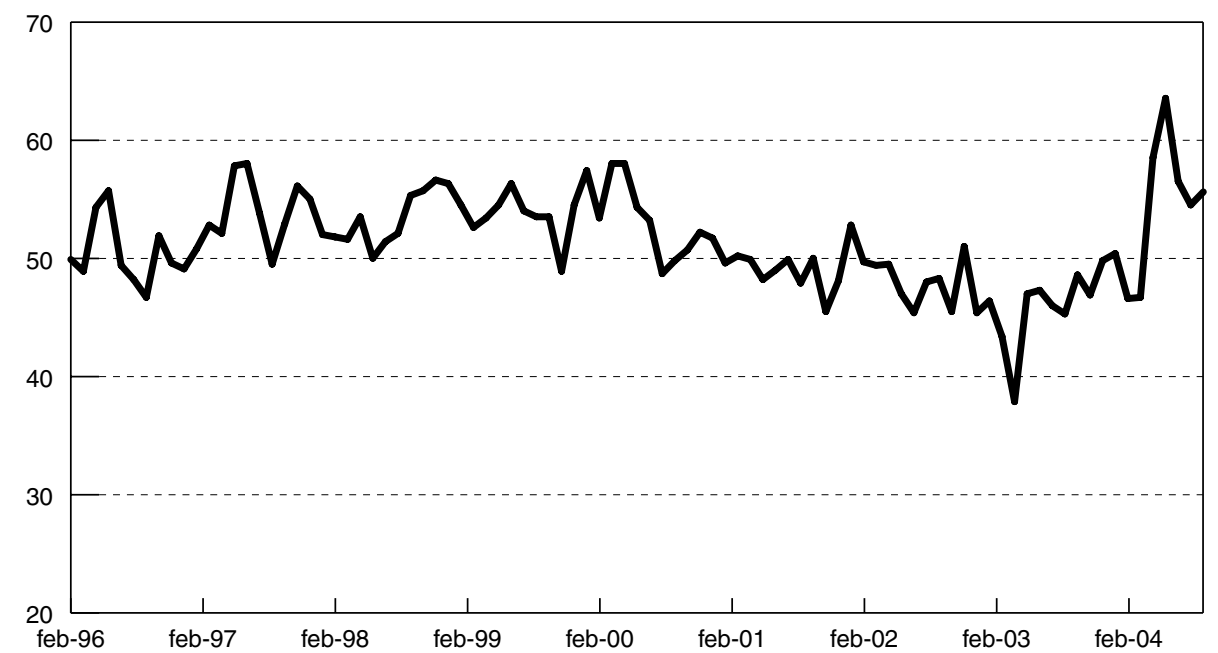

GRÁFICO 9

Indicador Situación Política Actual (SPA)

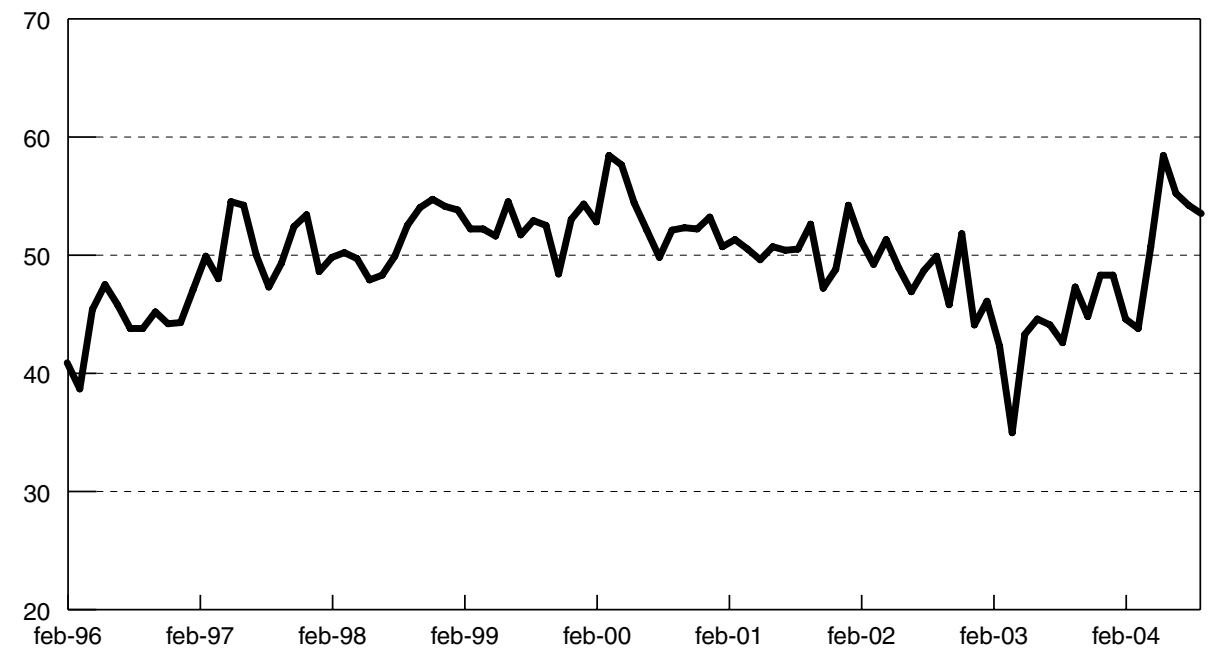


GRÁFICO 10

Indicador Expectativas Políticas (IEP)

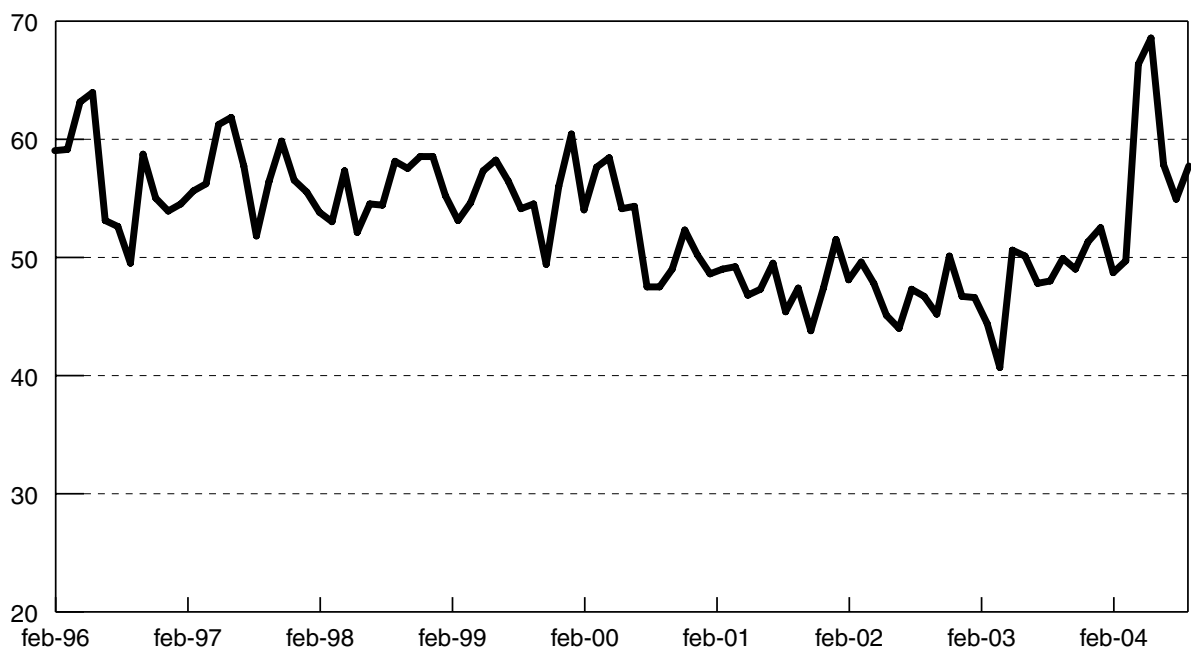

GRÁFICO 11

Indicadores de Situación Política

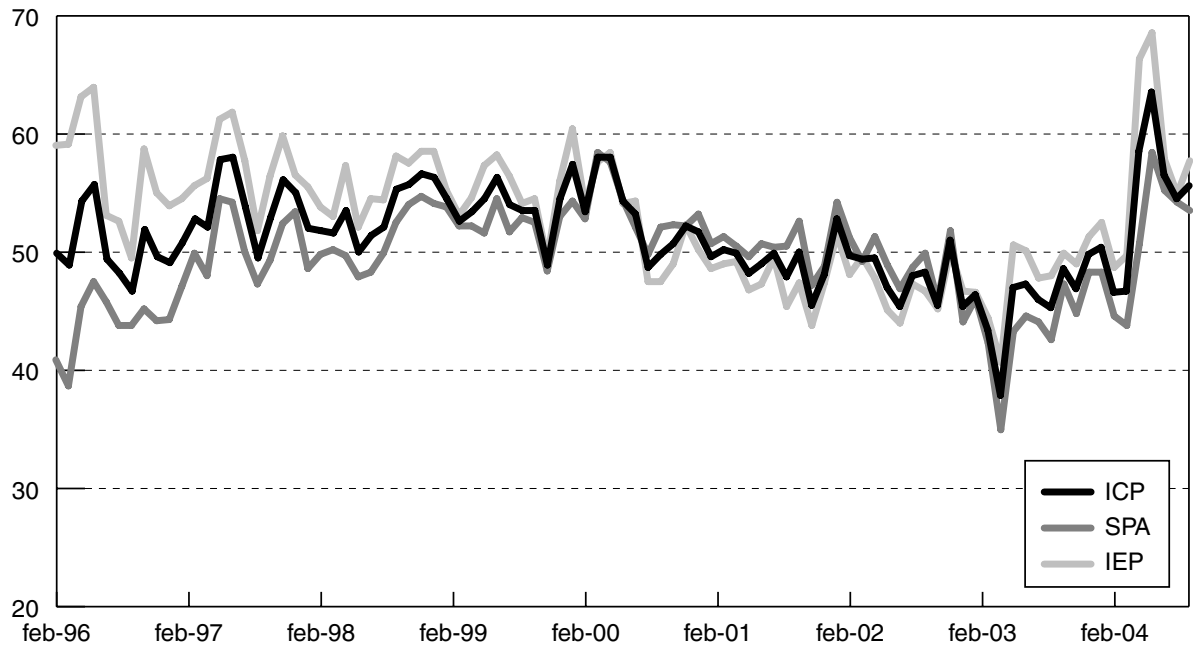




\section{INDICADORES DEL SISTEMA POLÍTICO}

Las preguntas de los barómetros que se consideran para estos indicadores son:

P1: En su conjunto, ¿cómo calificaría Ud. la gestión que está haciendo el gobierno del PSOE: muy buena, buena, regular, mala o muy mala?

P2: En su conjunto, ¿cómo calificaría Ud. la gestión que está haciendo el gobierno del PP: muy buena, buena, regular, mala o muy mala?

P3: En general, ¿cómo calificaría Ud. la actuación política que está teniendo el PSOE en la oposición: muy buena, buena, regular, mala o muy mala?

P4: En general, ¿cómo calificaría Ud. la actuación política que está teniendo el PP en la oposición: muy buena, buena, regular, mala o muy mala?

En los barómetros del primer mes de cada trimestre desde 1996 se realizan las preguntas P1 y P4 ó P2 y P3 alternativamente, dependiendo de cuál es el partido en el gobierno y cuál es el primer partido de la oposición.

A partir de estas preguntas se construyen, en primer lugar, las series de Valoración de la Labor del Gobierno y Valoración de la Labor del Primer Partido de la Oposición sin más que intercalar las preguntas P1 y P2, por un lado, y P3 y P4, por el otro, respectivamente (evalúan el comportamiento del gobierno y la oposición, independientemente del partido al que pertenezcan sus miembros).

Posteriormente se elaboran los indicadores:

- Indicador de la Labor del Gobierno (ILG):

$$
I L G=\frac{100 \cdot p_{1}+75 \cdot p_{2}+50 \cdot p_{3}+25 \cdot p_{4}+0 \cdot p_{5}}{p_{1}+p_{2}+p_{3}+p_{4}+p_{5}}
$$

donde $p_{1}, p_{2}, p_{3}, p_{4}$ y $p_{5}$ son, respectivamente, las proporciones de respuesta de las opciones muy buena, buena, regular, mala y muy mala, respectivamente, en las preguntas P1 y $\mathrm{P} 2$. 
- Indicador de la Labor de la Oposición (ILO):

$$
I L O=\frac{100 \cdot p_{1}+75 \cdot p_{2}+50 \cdot p_{3}+25 \cdot p_{4}+0 \cdot p_{5}}{p_{1}+p_{2}+p_{3}+p_{4}+p_{5}}
$$

donde $p_{1}, p_{2}, p_{3}, p_{4}$ y $p_{5}$ son, respectivamente, las proporciones de respuesta de las opciones muy buena, buena, regular, mala y muy mala, respectivamente, en las preguntas P3 y $\mathrm{P} 4$.

Se construye después el Indicador de Confianza del Sistema Político (ICSP) como la media aritmética de los otros dos:

$$
I C S P=\frac{I L G+I L O}{2}
$$

En los gráficos 12 a 15 se muestran estos indicadores.

GRÁFICO 12

Indicador de Confianza del Sistema Político (ICSP)

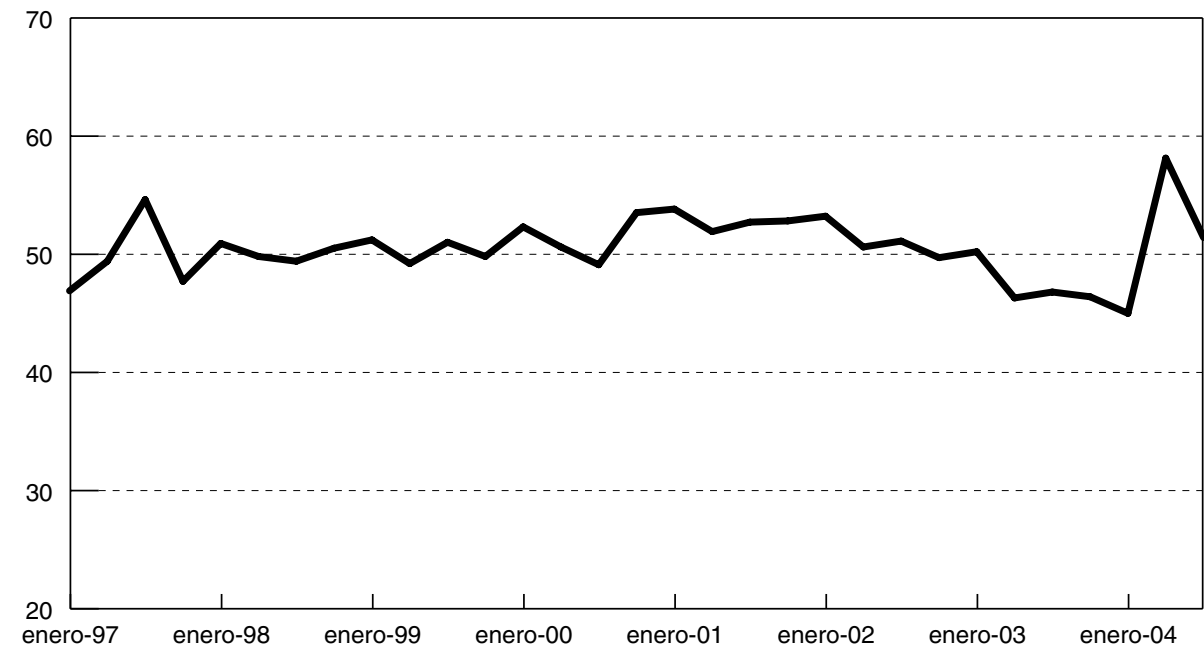


GRÁFICO 13

Indicador de la Labor del Primer Partido de la Oposición (ILO)

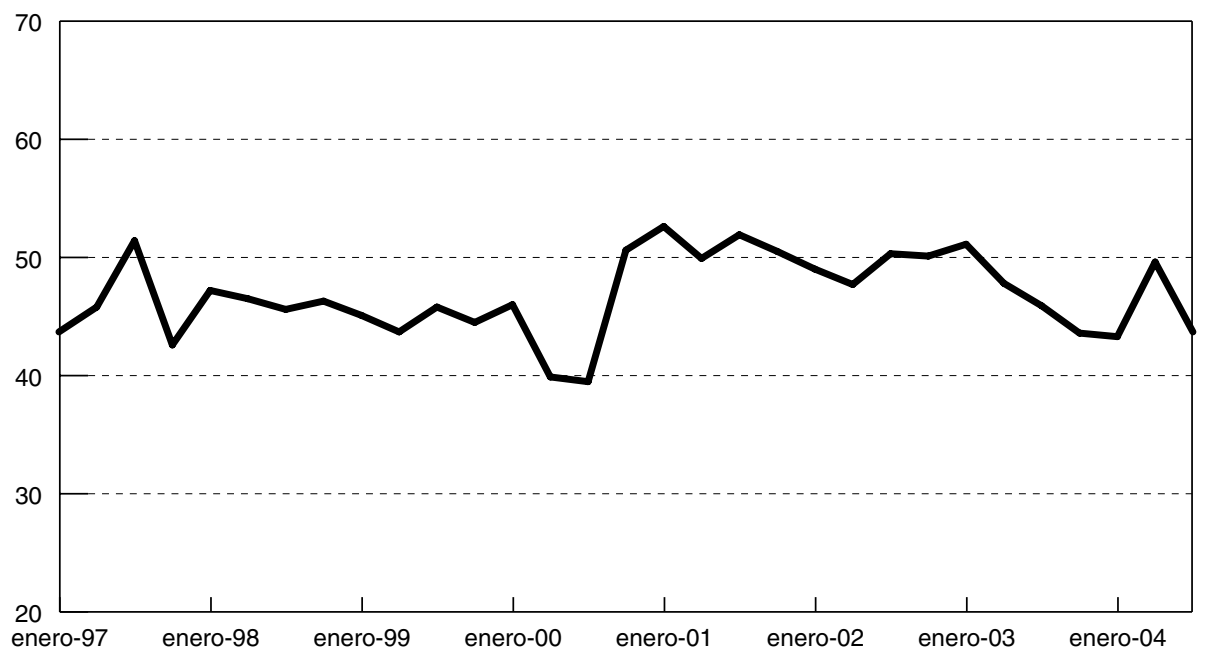

GRÁFICO 14

Indicador de la Labor del Gobierno (ILG)

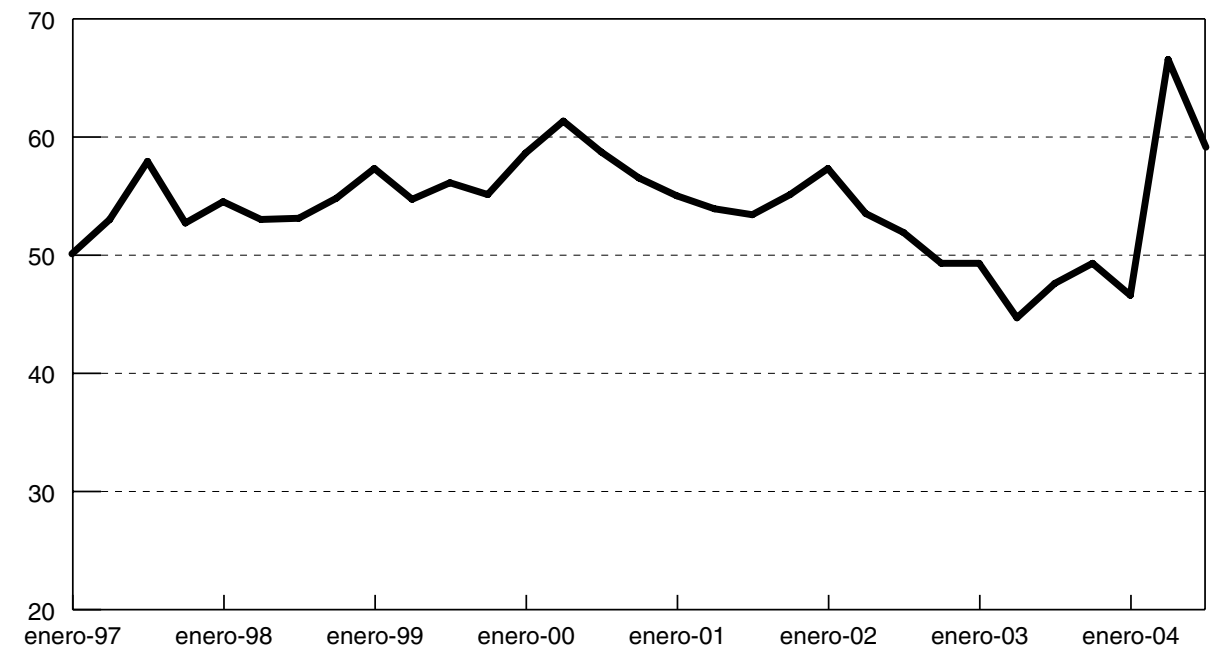


GRÁFICO 15

Indicadores del Sistema Político

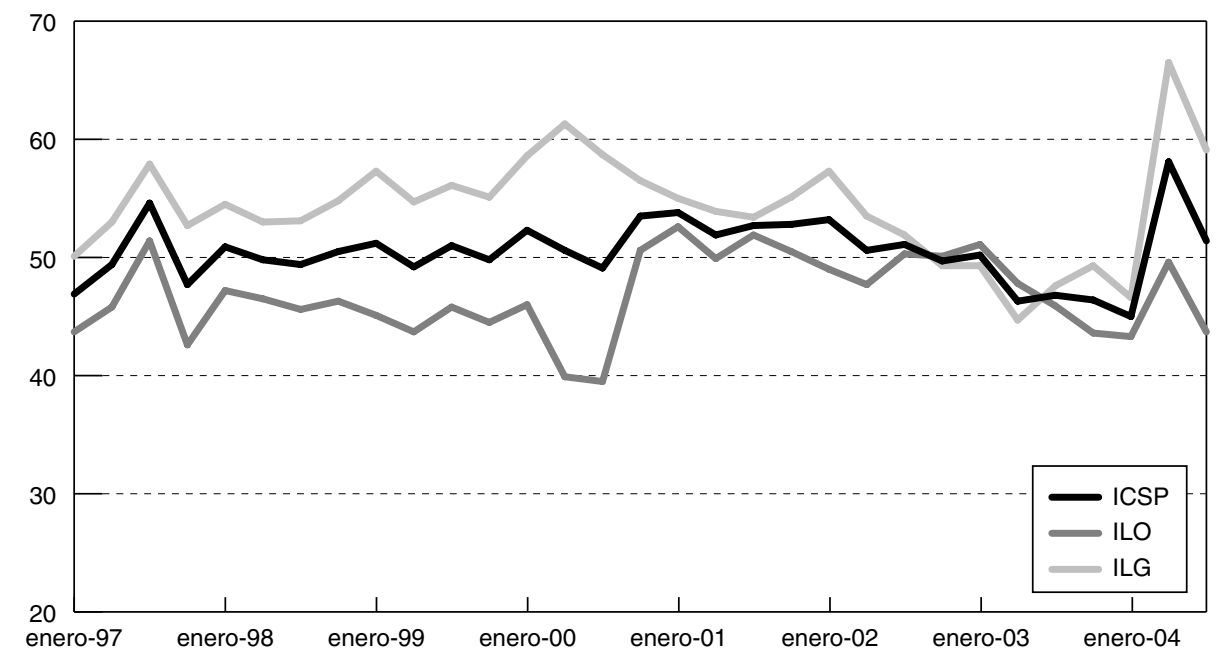

\section{INDICADORES ELECTORALES}

Las series electorales se obtienen de la siguiente pregunta de los barómetros del primer mes de cada trimestre:

Suponiendo que mañana se celebrasen elecciones generales, es decir, al Parlamento español, ¿a qué partido votaría Ud.?

El primer indicador se conoce como Intención de Voto, y no es más que la serie de los porcentajes de respuesta para cada partido político. Esta serie en sí misma constituye un indicador que no precisa de ninguna elaboración.

Se presentan los resultados para los principales partidos en los gráficos 16 y 17 . 
GRÁFICO 16

Intención de Voto (I)

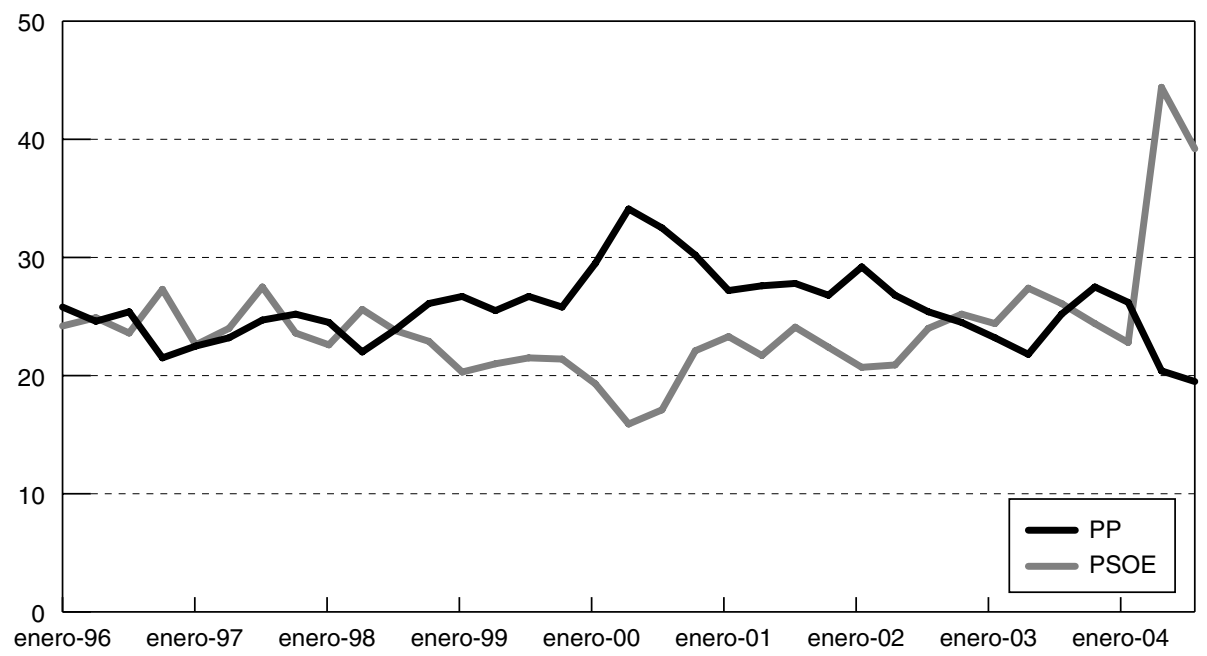

GRÁFICO 17

Intención de Voto (II)

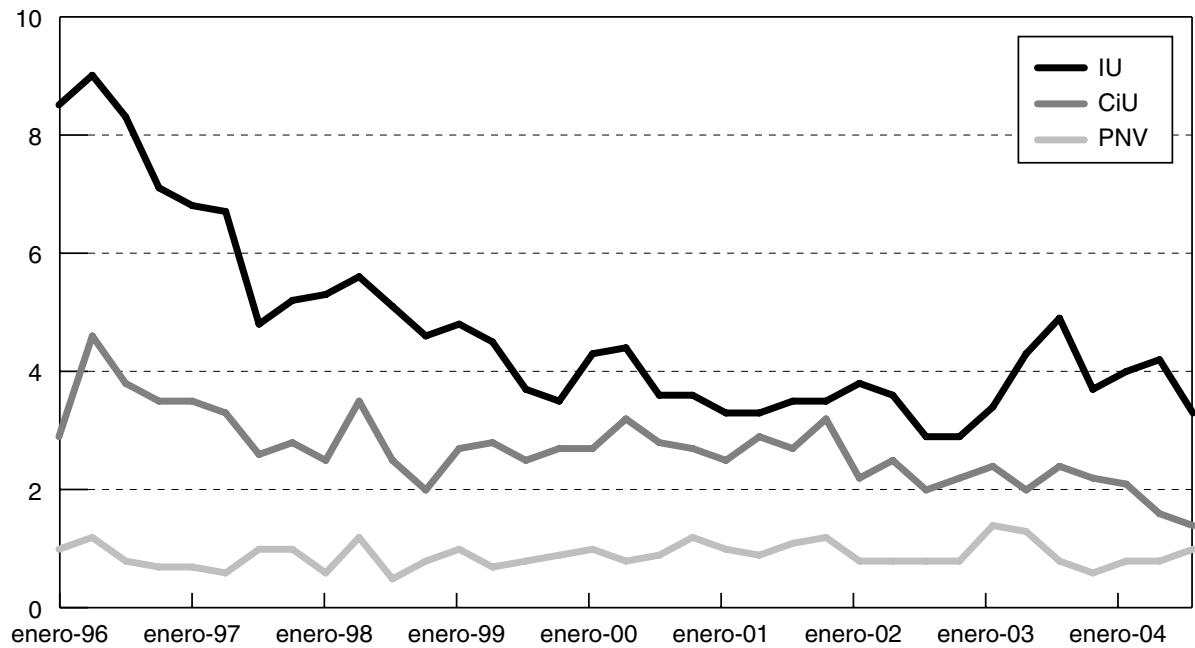


Sin embargo, las respuestas a esta pregunta no constituyen una buena previsión si lo que se pretende es acercarse a los resultados de unas elecciones próximas, ya que son muy altos los porcentajes de respuesta para las opciones No sabe y No contesta.

Para predecir mejor los resultados electorales, el CIS construye a partir de la Intención de Voto lo que se conoce como Estimación de Voto, que no es más que aplicar a la Intención de Voto un modelo de corrección basado en otras variables de la encuesta, la experiencia pasada, informaciones de tipo cualitativo, etc.

Por este motivo, la Estimación de Voto no es un indicador comparable al resto de los que se presentan, en la medida en que su método de cálculo nunca se ha hecho público y ha cambiado con los distintos equipos de dirección del CIS. Podría considerarse que no constituye una auténtica serie temporal, pero, por su interés, se presenta entre los indicadores electorales y se muestra en los gráficos 18 y 19 para los principales partidos.

GRÁFICO 18

Estimación de Voto (I)

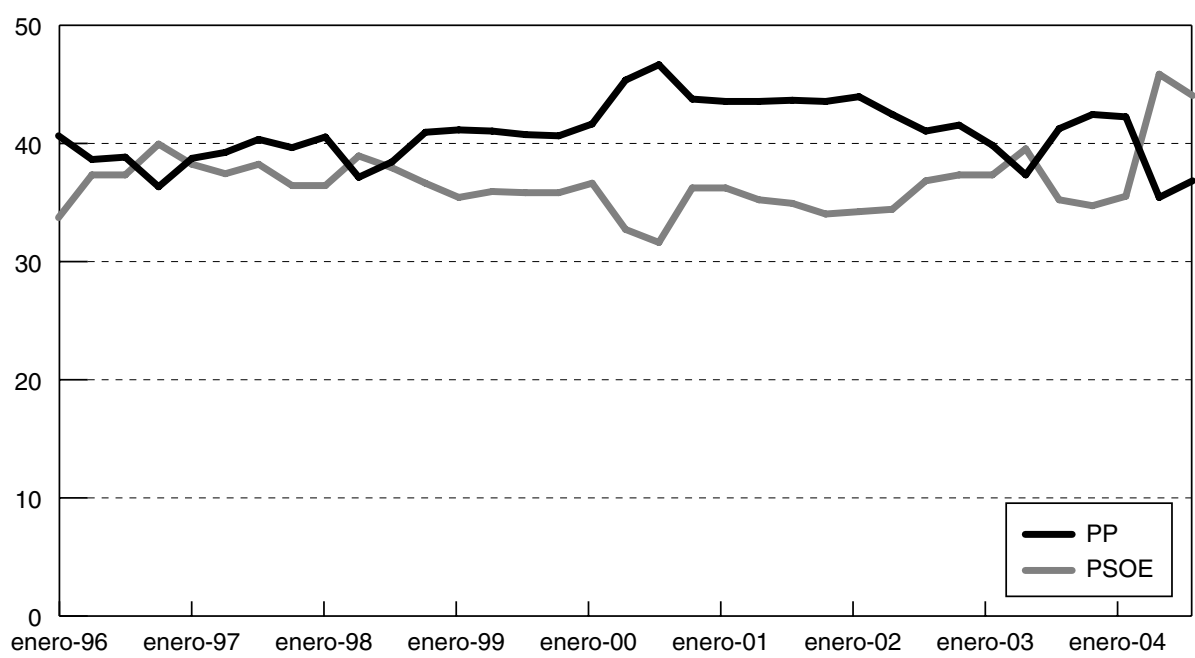


GRÁFICO 19

Estimación de Voto (II)

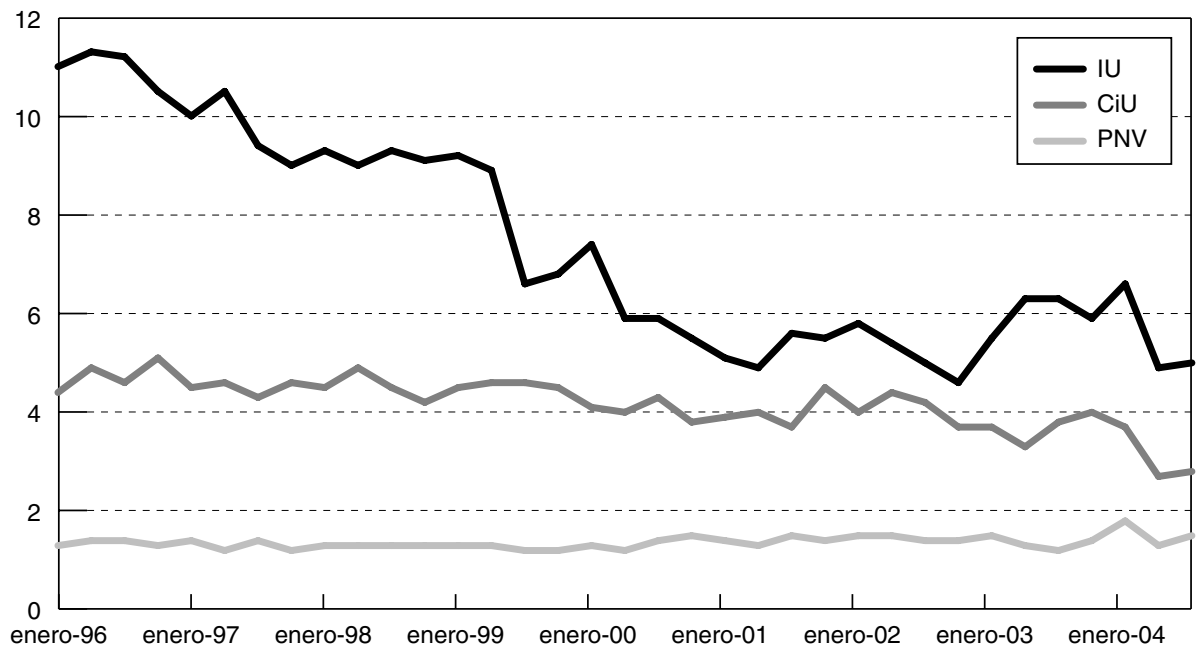

\section{AUTOUBICACIÓN IDEOLÓGICA}

A partir de la pregunta:

Cuando se habla de política se utilizan normalmente las expresiones izquierda y derecha. En esta tarjeta hay una serie de casillas que van de izquierda (con el valor 1) a derecha (con el valor 10). ¿En qué casilla se situaría Ud.?

se construye el indicador de Autoubicación Ideológica como la media aritmética de las respuestas (eliminando los No sabe y No contesta). Al ser una variable de tipo cuantitativo, la media es el más sencillo y mejor indicador a elegir.

Se suele calcular también la desviación típica, que se mantiene prácticamente constante, para verificar la validez del cálculo de la media.

En el gráfico 20 se muestra el indicador. 
GRÁFICO 20

Autoubicación Ideológica Media

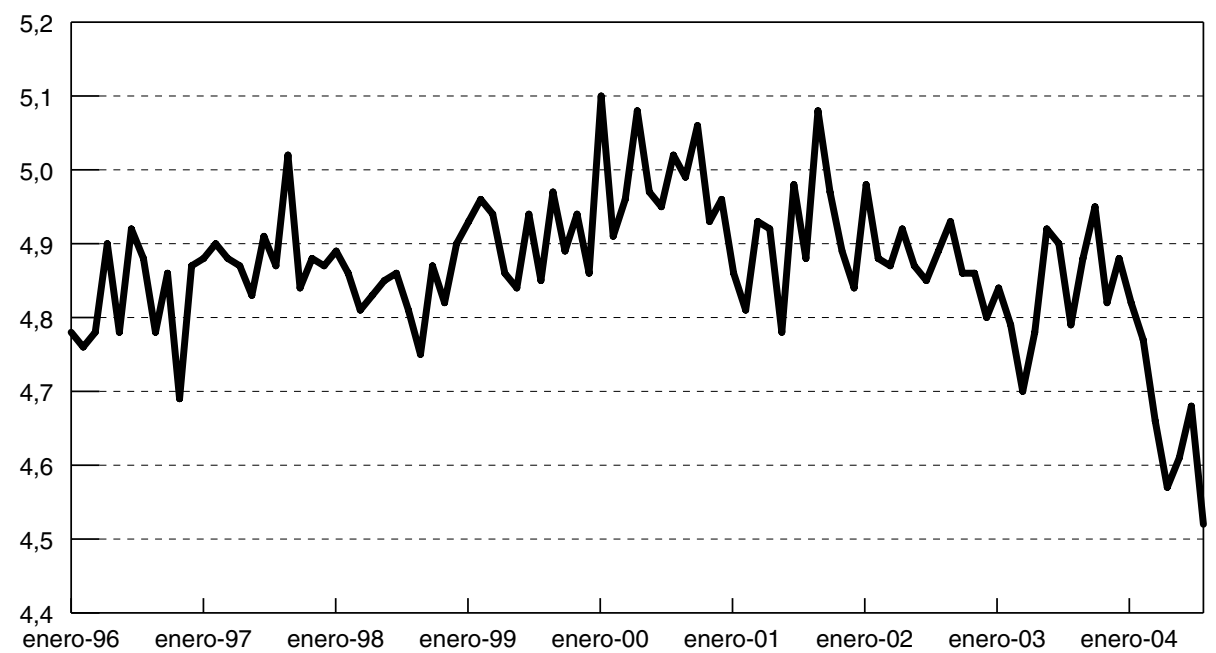

Es importante señalar que este indicador está sesgado a la izquierda en una medida difícil de evaluar. La utilización de la escala de 1 a 10 produce este efecto indeseado (probablemente se utiliza para evitar las connotaciones negativas de asignar la puntuación 0 a la izquierda).

En efecto, si se estudian los porcentajes de respuesta para cada una de las puntuaciones de 1 a 10 se observa que, sistemáticamente, el valor 5 constituye una moda muy alta en todas las fechas. Esto parece indicar que los respondentes interpretan el valor 5 como un valor medio, por estar habituados a manejar, en muchas situaciones, la escala del 0 al 10 en la que el valor 5 es el punto medio. Sin embargo, en la escala del 1 al 10 que se utiliza en esta pregunta el valor medio es el 5,5, por lo que la media aritmética obtenida estará más a la izquierda de lo que corresponde.

\section{BIBLIOGRAFÍA}

- Essays in Index Number Theory, W. E. Diewert, A. O. Nakamura. Ed. North-Holland (1993). 


\section{ABSTRACT}

Recently, a set of indicators drawn up using the monthly Opinion Barometers have started to be shown on the web page of the Centro de Investigaciones Sociologicas (Centre for Sociological Research). This note describes the methodology used for their construction, but before this, it shows other similar indicators drawn up in different institutions.

Key words: Postal Surveys, Telephone Surveys, Population Framework, Non-Coverage. 